Utah State University

DigitalCommons@USU

8-20-2014

\title{
Far-Infrared Spectroscopy of the Troposphere: Calibration with a Cold Background
}

\author{
Harri Latvakoski \\ Utah State University \\ Martin G. Mlynczak \\ Utah State University \\ Richard P. Cageao \\ Utah State University \\ David G. Johnson \\ Utah State University \\ David P. Kratz \\ Utah State University
}

Follow this and additional works at: https://digitalcommons.usu.edu/sdl_pubs

\section{Recommended Citation}

Latvakoski, Harri; Mlynczak, Martin G.; Cageao, Richard P.; Johnson, David G.; and Kratz, David P., "FarInfrared Spectroscopy of the Troposphere: Calibration with a Cold Background" (2014). Space Dynamics Lab Publications. Paper 81.

https://digitalcommons.usu.edu/sdl_pubs/81

This Article is brought to you for free and open access by the Space Dynamics Lab at DigitalCommons@USU. It has been accepted for inclusion in Space Dynamics Lab Publications by an authorized administrator of DigitalCommons@USU. For more information, please contact digitalcommons@usu.edu.

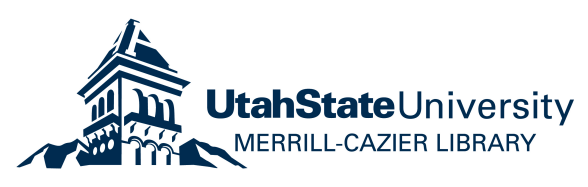




\title{
Far-infrared spectroscopy of the troposphere: calibration with a cold background
}

\author{
Harri Latvakoski, ${ }^{1}$ Martin G. Mlynczak, ${ }^{2, \star}$ Richard P. Cageao, ${ }^{3}$ \\ David G. Johnson, ${ }^{3}$ and David P. Kratz ${ }^{2}$ \\ ${ }^{1}$ Space Dynamics Laboratory, 1695 North Research Park Way, North Logan, Utah 84341, USA \\ ${ }^{2}$ Science Directorate, NASA Langley Research Center, Mail Stop 420, Hampton, Virginia 23681, USA \\ ${ }^{3}$ Engineering Directorate, NASA Langley Research Center, Mail Stop 468, Hampton, Virginia 23681, USA \\ ${ }^{*}$ Corresponding author: m.g.mlynczak@ nasa.gov
}

Received 28 April 2014; accepted 4 June 2014;

posted 17 July 2014 (Doc. ID 207888); published 15 August 2014

\begin{abstract}
The far-infrared spectroscopy of the troposphere (FIRST) instrument is a Fourier-transform spectrometer developed to measure the Earth's thermal emission spectrum with a particular emphasis on the far-infrared. FIRST has observed the atmosphere from both the ground looking up and from a highaltitude balloon looking down. A recent absolute laboratory calibration of FIRST under ground-like operating conditions showed accuracy to better than $0.3 \mathrm{~K}$ at near-ambient temperatures (270-325 K) but reduced accuracy at lower temperatures. This paper presents calibration results for balloon-flight conditions using a cold blackbody to simulate the space view used for on-board calibration. An unusual detector nonlinearity was discovered and corrected, and stray light was measured and removed. Over most of the range of Earth scene temperatures (205-300 K), the accuracy of FIRST is 0.35-0.15 K (one sigma). (C) 2014 Optical Society of America

OCIS codes: (010.0010) Atmospheric and oceanic optics; (010.0280) Remote sensing and sensors; (010.7030) Troposphere.

http://dx.doi.org/10.1364/AO.53.005425
\end{abstract}

\section{Introduction}

Over the past 20 years, the importance of direct farinfrared (far-IR) measurements of Earth's emission spectrum has become widely recognized. The farIR contains approximately one half of the Earth's outgoing longwave radiation and greenhouse effect [1]; it is responsible for almost the entire radiative cooling of the free troposphere [2]; and cirrus clouds exert a strong radiative effect in the far-IR [3]. Harries et al. [4] summarize the importance of the far-IR to the Earth's climate system.

The far-infrared spectroscopy of the troposphere (FIRST) instrument was developed under NASA's Instrument Incubator Program to demonstrate

1559-128X/14/245425-09\$15.00/0

(C) 2014 Optical Society of America technology (in a space-like environment) to measure the far-IR spectrum $\left(15-100 \mu \mathrm{m} ; 650-100 \mathrm{~cm}^{-1}\right)$. FIRST was built by the Space Dynamics Laboratory (SDL) in Logan, Utah, in collaboration with the NASA Langley Research Center. Since it was built in 2005, FIRST has been used to observe the atmosphere from both the ground and from high-altitude balloons $[5,6]$.

In 2011, the FIRST instrument was returned to SDL for absolute radiance recalibration. That calibration was performed for the ground-based observation mode of FIRST, which uses a warm and an ambient blackbody for on-board calibration and showed FIRST to be accurate in the far-IR to better than $0.3 \mathrm{~K}$ for $270-325 \mathrm{~K}$ scene temperatures, $0.5 \mathrm{~K}$ at $247 \mathrm{~K}$ and $1 \mathrm{~K}$ at $225 \mathrm{~K}$ [7]. The reduced accuracy at lower temperatures is due to magnification of errors when extrapolating away from the temperatures 
of the two calibrator blackbodies. In 2013, FIRST was recalibrated for absolute accuracy in its balloonbased operating mode. During balloon operation, FIRST uses a warm blackbody (WBB) and a view of cold space for on-board calibration, instead of a WBB and an ambient blackbody, so different performance was expected (e.g., extrapolation is no longer an issue). During lab calibration, the space view is simulated by a cold blackbody source. This paper describes the results of the cold background calibration of FIRST performed in 2013. The lessons learned from this calibration activity and from the prior warm source calibration activity [7] will be extremely valuable in developing space-flight missions designed to measure the far-IR spectrum [8].

The instrument is described in Section 2. The calibration equipment and testing are described in Section 3. The calibration results are described in Section $\underline{\overline{4}}$. Section $\underline{5}$ is the conclusion.

\section{FIRST Instrument Description}

Table 1 lists the basic FIRST instrument parameters. A complete description of the instrument and basis for the design is provided elsewhere [7,9]. The noiseequivalent temperature difference $(\overline{\mathrm{N}} \overline{\mathrm{E}} \Delta \mathrm{T})$ in the table is for a single spectrum of a target. The absolute accuracy goal of FIRST was established to be identical to the precision, acknowledging the intent to average many spectra to substantially reduce random noise in the overall error budget. The prior calibration [7] and this calibration demonstrate that FIRST does meet these goals for scene temperatures of $\sim 270-325 \mathrm{~K}$.

The FIRST instrument (Fig. 1) consists of three sections separated by vacuum windows: the sceneselect assembly, the interferometer section, and the detector dewar. The vacuum windows are $41 \mu \mathrm{m}$ thick polypropylene. The window between the detector dewar and interferometer section is a single sheet while the window between the interferometer section and the scene-select assembly is two sheets in series for increased strength. The detector dewar contains

\section{Table 1. FIRST Instrument Parameters}

\begin{tabular}{ll}
\hline Instrument type & $\begin{array}{c}\text { Fourier transform spectrometer } \\
\text { (Michelson interferometer) }\end{array}$ \\
Spectral range & $100-1000 \mathrm{~cm}^{-1}($ with some coverage \\
& $\left.50-2200 \mathrm{~cm}^{-1}\right)$ \\
Resolution & $0.643 \mathrm{~cm}^{-1}$ \\
Scan time & $11.5 \mathrm{~s}$ \\
Interferogram size & 24576 points \\
Aperture & $7 \mathrm{~cm}$ \\
Focal ratio & f 6.5 \\
Beamsplitter & Ge on thin polypropylene film \\
Detectors & Ten $(10)$ Liquid He cooled Si bolometers \\
On board calibration & Two on-board blackbodies or one \\
& blackbody and a space view \\
Field of view & Ten $0.41^{\circ} \mathrm{FOV}$ detectors in a sparsely \\
& populated $4.4^{\circ} \times 4.4^{\circ} \mathrm{FOV}$ \\
NE $\Delta \mathrm{T}$ & $0.2 \mathrm{~K}, 170-960 \mathrm{~cm}^{-1} ; 0.5 \mathrm{~K}$ \\
& $100-170 \mathrm{~cm}^{-1}($ performance goal) \\
\hline
\end{tabular}

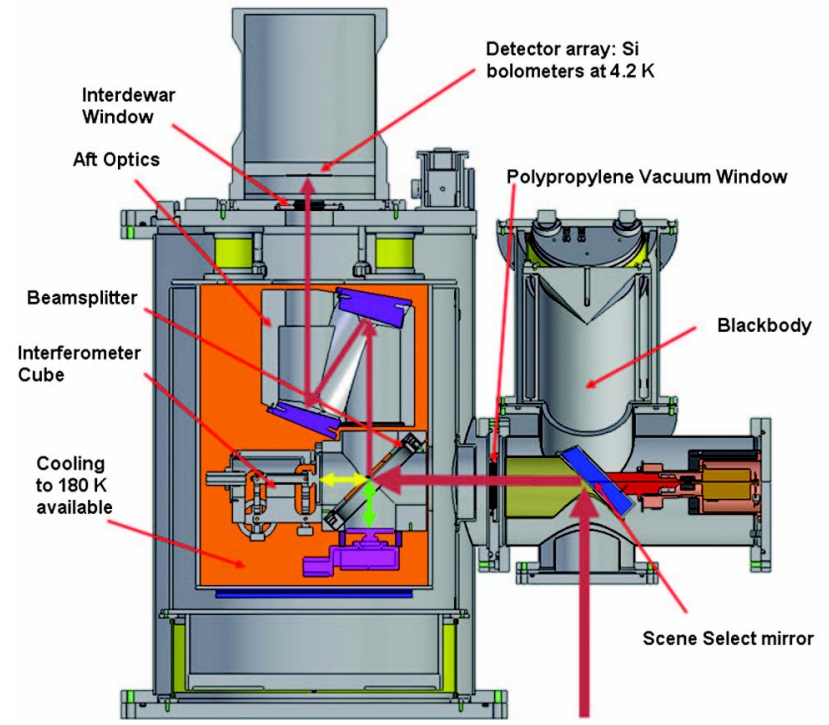

Fig. 1. Cutaway view of FIRST with the principal components labeled and the light path shown by the thick red arrows.

the 10 detectors and cryogen tanks. The interferometer section contains the interferometer and the aft optics that focus the beam from the interferometer onto the detectors. The scene-select assembly contains a rotating mirror that directs the incoming beam to one of three ports: the instrument aperture or one of two calibration ports. The entire sceneselect assembly can be rotated where it attaches to the interferometer section so that an open port can face up (for observations from the ground) or down (for observations from a balloon) or to the side (for calibration in the laboratory).

\section{FIRST Ground Calibration}

FIRST was calibrated for absolute radiance in the balloon-observing mode by having the on-board WBB attached to one port of the scene-select assembly, a space view simulator (SVS) blackbody on the second port, and the long wave infrared calibration source (LWIRCS) [7,10,11] blackbody on the third port of the scene-select assembly. LWIRCS (Table 2 , Fig. 2) is a ground calibration blackbody of a similar design to the FIRST on-board blackbodies. The output of LWIRCS has been measured with the National Institute of Standards and Technology (NIST) transfer radiometer (TXR) [12], which confirms the performance of LWIRCS in the TXR bands (5 and $10 \mu \mathrm{m}$ ) to the uncertainty level of the TXR (approximately $90 \mathrm{mK}$ at $5 \mu \mathrm{m}$ and about $150 \mathrm{mK}$ at $10 \mu \mathrm{m})$ [7]. These calibrations were performed at SDL in March 2013.

The FIRST instrument is calibrated with the LWIRCS by setting the LWIRCS to a specific temperature and conducting a data-collection sequence, in which the SVS is observed followed by the WBB, LWIRCS, and then the SVS and WBB again. Each blackbody was observed for a few minutes, with LWIRCS observed for about twice as long as the others. LWIRCS temperatures were in the range of 
Table 2. Performance Specifications for the LWIRCS Blackbody

\begin{tabular}{lc}
\hline Wavelength range & $1-100 \mu \mathrm{m}$ \\
Temperature range & $90-350 \mathrm{~K}$ \\
Aperture & $15.5 \mathrm{~cm}$ \\
Beam divergence & $6^{\circ}$ full angle \\
$\quad$ accepted & \\
Emissivity & $\geq 0.9998(1-35 \mu \mathrm{m}) ; \geq 0.9980(35-100 \mu \mathrm{m})$ \\
Temperature & $130 \mathrm{mK}$ at $180 \mathrm{~K}, 60 \mathrm{mk}$ at $290 \mathrm{~K}$ \\
$\quad$ uncertainty & \\
\hline
\end{tabular}

$150-325 \mathrm{~K}$. In all cases, the SVS was cooled to $77 \mathrm{~K}$, and the WBB was held at $324 \mathrm{~K}$.

A general inverse fast Fourier transform algorithm and associated processing is used to convert the FIRST interferograms into spectra. A few atypical steps are required to process FIRST data [7], among them the handling of data affected by vibration. For the most part, the effects of vibration are at acceptable levels, but occasionally, outside sources add excessive vibration to some interferograms that must be excluded. Some effects of vibration are visible in the calibration data. These are identifiable because they are more prominent at a high wavenumber and are independent from scan to scan but affect spectra from all detectors in the same way.

For each detector, the resulting good spectra were averaged for each target with separate averages kept for each direction of the mirror movement. The six (forward and backward spectra for the WBB, SVS, and LWRICS) resulting average spectra per detector for each data set are complex numbers. Figure $\underline{3}$ shows averaged spectra of the SVS, WBB, and LWIRCS in one scan direction.

No detector nonlinearity was observed during the previous calibration [7]. Detector nonlinearity produces artifacts in the spectrum realized from a transformed interferogram, with a broad hump of increased signal near-zero wavenumber the most consistent and obvious artifact, regardless of the functional form of the nonlinearity. The lack of such artifacts in the spectra was consistent with any nonlinearity being less than $\sim 0.3 \%$ at the peak of

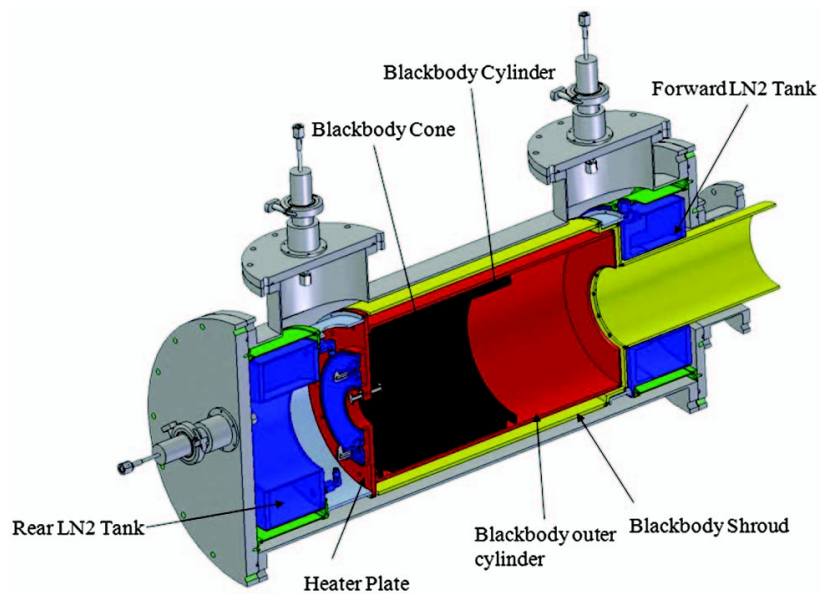

Fig. 2. Cutaway view of the LWIRCS blackbody.

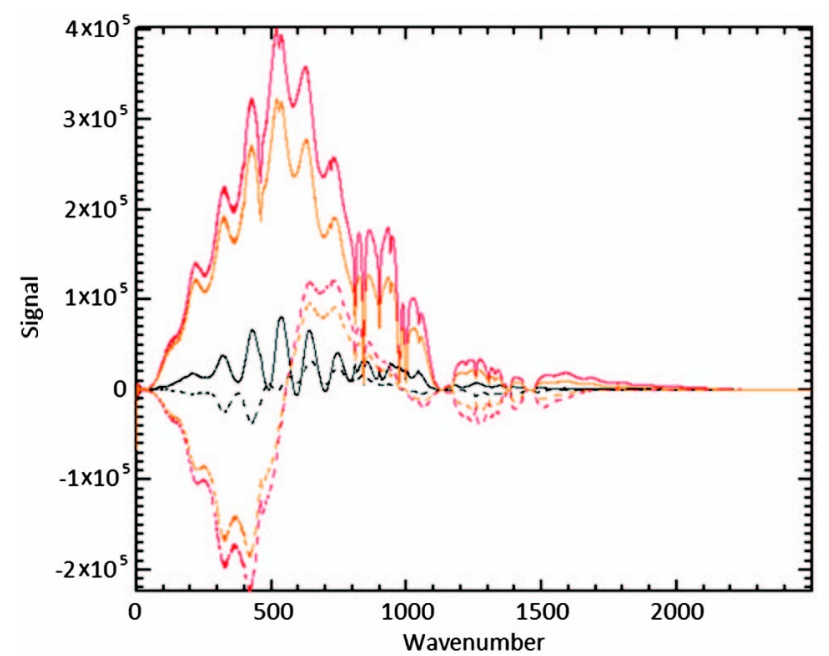

Fig. 3. Spectra of the SVS (black), WBB (red), and LWIRCS at $292 \mathrm{~K}$ (orange) from detector 2 at full $0.6428 \mathrm{~cm}^{-1}$ resolution. The real part is the solid line and the imaginary part is the dashed line.

the interferogram. The cold-source spectra show a similar result; the spectra indicate no nonlinearity in the interferograms above the $\sim 0.3 \%$ level at the interferogram peak. However, an unusual form of detector nonlinearity was subsequently discovered and this is discussed in Section 4 .

The FIRST spectra are converted to radiances using the calibration equation for FIRST:

$$
P_{\text {Target }}=\frac{S_{\text {Target }}-S_{\mathrm{SVS}}}{S_{\mathrm{WBB}}-S_{\mathrm{SVS}}}\left(P_{\mathrm{WBB}}-P_{\mathrm{SVS}}\right)+P_{\mathrm{SVS}}
$$

$P_{\text {Target }}$ is the target radiance, $S_{\text {Target }}$ is the signal measured by FIRST viewing the target under observation (LWIRCS during ground calibration), $\mathrm{S}_{\mathrm{WBB}}$ and $\mathrm{S}_{\mathrm{SVS}}$ are the signals measured by FIRST viewing the WBB and SVS, and $\mathrm{P}_{\mathrm{WBB}}$ and $\mathrm{P}_{\mathrm{SVS}}$ are the radiances of the WBB and SVS.

\section{FIRST Absolute Calibration Results}

The LWIRCS brightness temperature is calculated from the FIRST LWIRCS radiances using the inverse Planck function. No correction is made for blackbody emissivity being less than 1.0 because the difference is not significant when assessing the overall uncertainty of the FIRST instrument. The brightness temperatures measured by FIRST when observing LWIRCS are compared with temperatures from the LWIRCS temperature sensors to assess the absolute calibration of FIRST.

Figure 4 shows the brightness temperature measured by FIRST with LWRICS at four temperatures. (Data were collected at nine temperatures from 150 to $325 \mathrm{~K}$, but the four in the figure are representative of all the results.) One obvious noise component in the brightness temperature is the rapid variation from spectral bin to spectral bin. The noise increases at high wavenumbers, low wavenumbers, and any other regions where FIRST response or signal is 

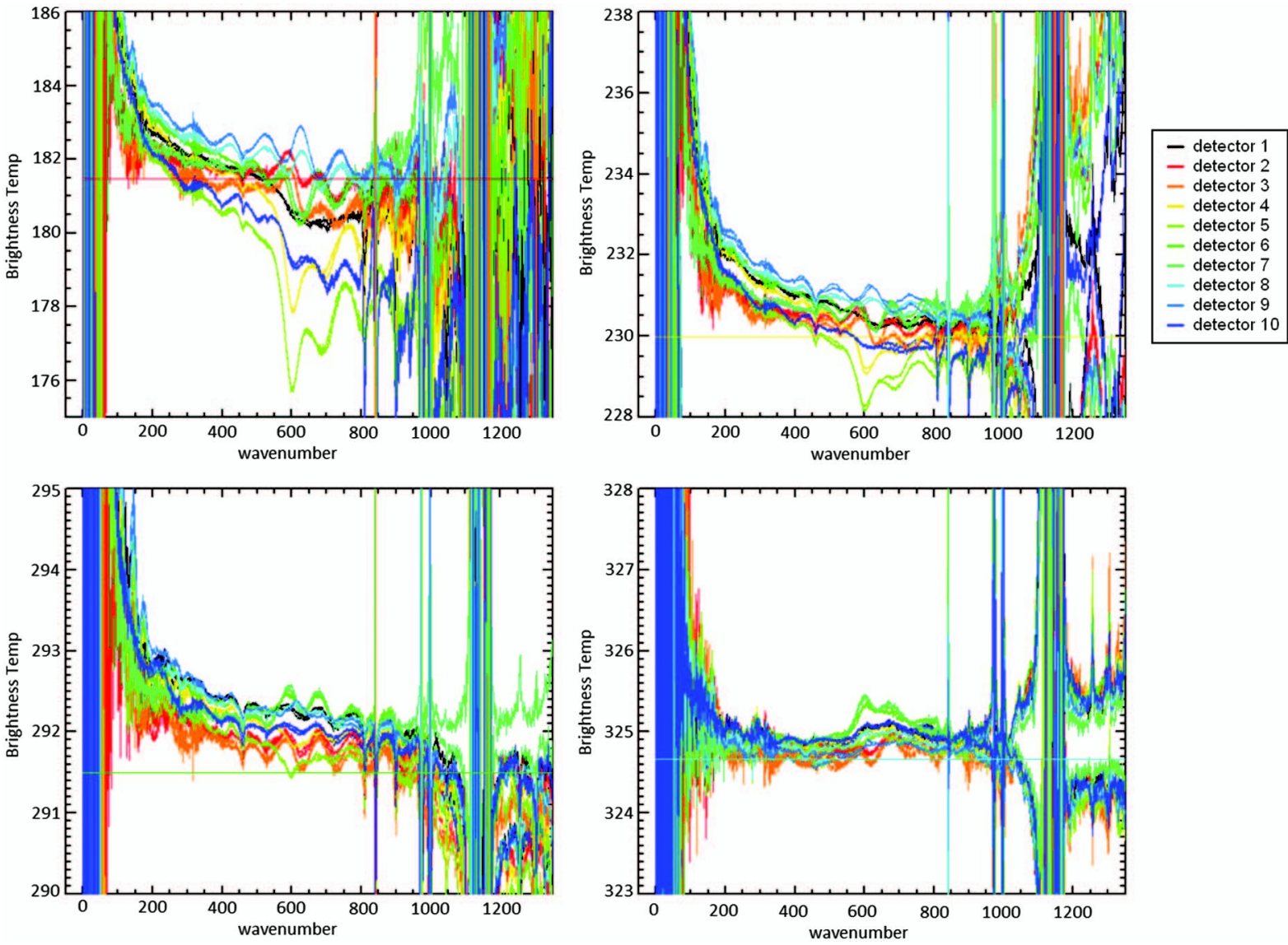

Fig. 4. Brightness temperature for LWIRCS as measured by each FIRST detector with LWIRCS at 181.48, 229.98, 291.49, and 324.66 K. On each plot the horizontal line is the actual temperature of LWIRCS during the data collection. Data from both scan directions are shown in the same color so there are two curves of each color. The resolution is $0.6428 \mathrm{~cm}^{-1}$.

low. This is random instrument noise, which increases notably at higher wavenumbers for colder temperature due to the rapid fall-off in signal with temperature there. The brightness temperatures for the two mirror-travel directions mainly trace each other to within the random noise but diverge at high wavenumbers, especially for the $325 \mathrm{~K}$ case. This divergence is due to vibration effects since it is different for the two scan directions but affects all detectors the same way.

Apart from these noise components, there are clear, highly systematic deviations between the brightness temperature and actual LWIRCS temperature, ranging from $\sim 0.5 \mathrm{~K}$ at $325 \mathrm{~K}$ (approximately the WBB temperature) with deviation increasing at low temperatures. The deviation is up to $6 \mathrm{~K}$ (peak) at $182 \mathrm{~K}$, but a few detectors are significantly worse than the rest. Excluding the three worst detectors (4, 5 , and 10 ), the peak deviation is $\sim 2 \mathrm{~K}$ at $182 \mathrm{~K}$. Some detectors show lower deviation; the detector 2 (red curve) result is always within $1 \mathrm{~K}$ of the actual LWIRCS temperature. The brightness temperatures were found to be very repeatable. For various LWIRCS temperatures, data taken several days apart with LWIRCS at nearly the same temperature had deviation from actual temperature that differed by no more than $0.2 \mathrm{~K}$ (peak deviation).
During the previous calibration [7], stray light was found to be a source of error. The sensitivity to stray light varied with detector, and those with high sensitivities during the previous calibration (4, 5, and 10) have the largest deviations in Fig. 4. The stray light can be quantified because LWIRCS has a baffle toward the front (the yellow cylinder at right in Fig. 3) that can be independently temperature controlled. By design, all FIRST detectors should not see light from this baffle, but keeping LWIRCS at a constant temperature and changing the baffle temperature shows a change in response, so the detectors, including those at the instrument detector array center (1 and 10), do see the baffle to varying degrees (Fig. 5). Since this stray light sensitivity was known, the baffle was kept at the same temperature as LWIRCS during this calibration, except when the stray light sensitivity was being tested. Because the baffle temperature is measured, the faction of the light coming from the baffle can be approximately calculated (Fig. 6). Given the blackbody apertures and locations, if FIRST is sensitive to light from this baffle, it is also likely to see stray light when viewing the other blackbodies or when viewing the atmosphere, though the amount will not be the same as from the LWIRCS baffle. 


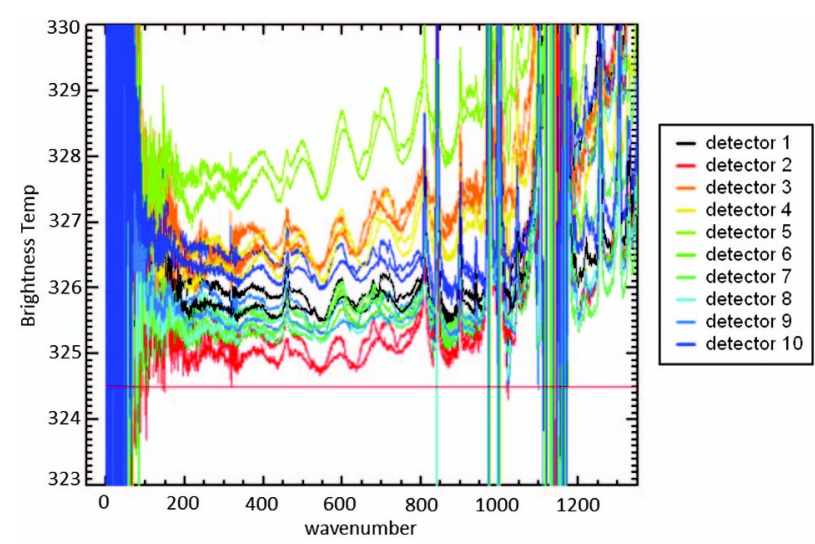

Fig. 5. Brightness temperature for LWIRCS at $324.49 \mathrm{~K}$ with the LWIRCS front baffle at $341.8 \mathrm{~K}$. Compare with the bottom right plot in Fig. 4 where LWIRCS is at $324.66 \mathrm{~K}$ and the baffle is at $321.4 \mathrm{~K}$.

One contributor to the stray light was found to be the vacuum window between the interferometer dewar and scene-select assembly. The amount of stray light (the fraction of light from the LWIRCS baffle) was observed to vary somewhat from vacuum cycle to vacuum cycle. This occurs because the window material is flexible, bows under differential pressure, and the window surface is uneven without pressure. During ground calibration, there is a vacuum on either side, and the two plastic films that comprise the window relax to some undefined shape, which can change with each vacuum cycle, and thus the window can direct the beam differently with each vacuum cycle. Figure 7 shows the LWIRCS brightness temperature as measured by FIRST at several temperatures with this window removed, and Fig. 8 shows the view fraction of the LWIRCS baffle calculated from data taken with the window removed. Comparing Figs. 6 and 8 shows that removing the window clearly reduces the stray light. (In particular, the stray light for detectors 3 and 4 is reduced to be similar to most others detectors.) It also removes oscillations in the data with a period of $\sim 100 \mathrm{~cm}^{-1}$, but stray light is still present. The elimination of

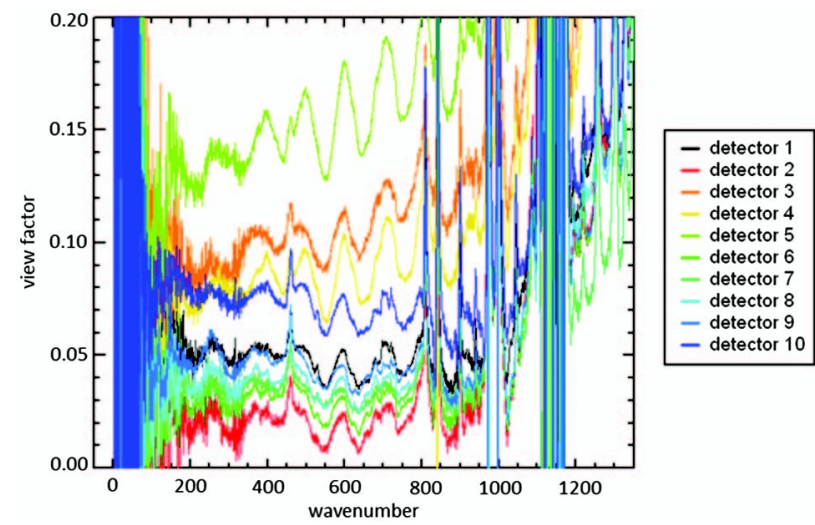

Fig. 6. Fraction of light seen by FIRST coming from the LWIRCS baffle as calculated from the data in Fig. $\underline{5}$. the oscillations shows the window's ability to direct the beam is wavenumber dependent.

Despite the change in stray light, the deviations shown in Figs. 4 and 7 are essentially the same magnitude except for the detectors that showed high stray light sensitivity when the window was present and show significantly different sensitivity without the window. This suggests the stray light is not the main cause of the deviations. This was confirmed by attempting to correct for stray light. Stray light affects the calibration equation by adding a contaminating radiance of $R$ with a view factor $f$ to each blackbody. The calibration equation [Eq. (1)] becomes

$$
\begin{aligned}
\left(1-f_{T}\right) P_{T}+f_{T} R_{T}= & x\left(\left(1-f_{W}\right) P_{W}+f_{W} R_{W}\right. \\
& \left.-\left(1-f_{S}\right) P_{S}-f_{S} R_{S}\right) \\
& +\left(1-f_{S}\right) P_{S}+f_{S} R_{S} .
\end{aligned}
$$

The subscripts $T, W$, and $S$ refer to the target (LWIRCS), the WBB, and SVS, respectively, and $x$ is the ratio of signals

$$
x \equiv \frac{S_{T}-S_{S}}{S_{W}-S_{S}} .
$$

Rearranging and combining terms reduces Eq. (2) to

$$
P_{T}=x\left(P_{W}-P_{C}+R_{1}\right)+P_{C}+R_{2} .
$$

This is just the calibration equation with two correcting radiances $R_{1}$ and $R_{2}$, which are combinations of the contaminating radiances and view factors:

$$
\begin{aligned}
& R_{1}=\frac{\left(f_{T}-f_{W}\right) P_{W}+f_{W} R_{W}-\left(f_{T}-f_{S}\right) P_{S}+f_{S} R_{S}}{1-f_{T}} \\
& R_{2}=\frac{\left(f_{T}-f_{S}\right) P_{S}+f_{S} R_{S}-f_{T} R_{T}}{1-f_{T}} .
\end{aligned}
$$

These correcting radiances are constant as long as the configuration does not change; therefore the effect of stray light can be corrected by including two correcting radiances into the calibration equation. Equation ( $\underline{3})$ can be rewritten as

$$
P_{T}-P_{C}-x\left(P_{W}-P_{C}\right) \equiv y=x R_{1}+R_{2}
$$

This is just a line with a slope and intercept of $R_{1}$ and $R_{2}$. Given data of LWIRCS at multiple temperatures, $R_{1}$ and $R_{2}$, can be found using line fits to the specified quantities at all wavenumbers.

In trying to apply such a fit to the LWIRCS brightness temperature data taken without a window, the plots of $x$ versus $y$ as defined in Eq. (6) are curves instead of straight lines (Fig. 9). If the correcting radiances found from this are applied to the data, the deviations improve somewhat, but clear systematic error remains, as may be expected when fitting a line to a curve. 

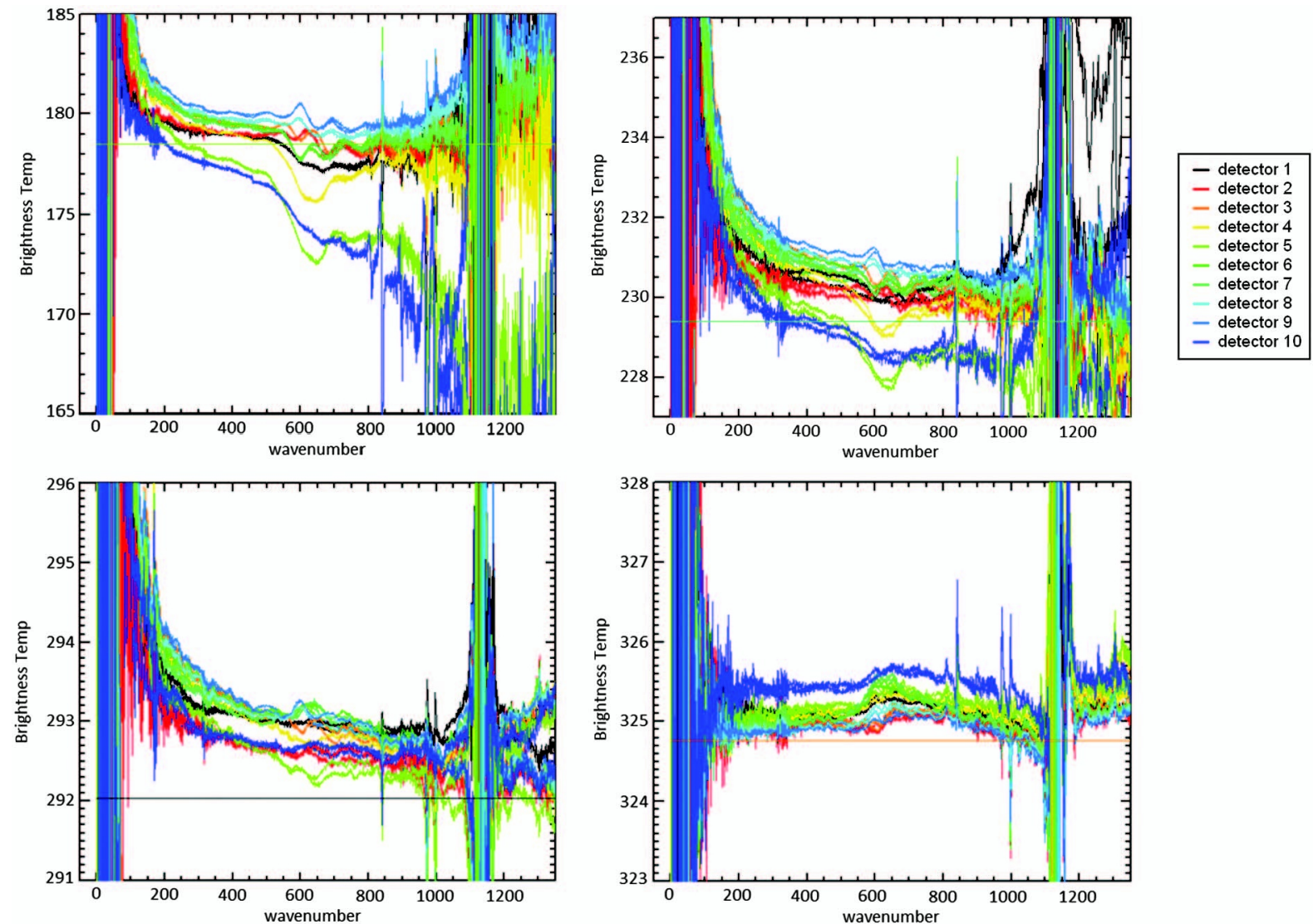

Fig. 7. Brightness temperature of LWIRCS at temperatures of 178.54, 229.39, 292.04, and $324.76 \mathrm{~K}$ with the window between the sceneselect assembly and interferometer dewar removed.

The source of the observed deviation was found to be a somewhat unusual form of detector nonlinearity. The bolometers in FIRST are read out by applying a constant current to the bolometer and reading out the voltage across the bolometer. When the target changes, the DC voltage across the bolometer changes as well (Fig. 10). This is not unexpected, but given the readout circuit, the change in voltage is proportional to a change in bolometer resistance, and the gain of a bolometer is known to be proportional to resistance [13]. Therefore, the spectra

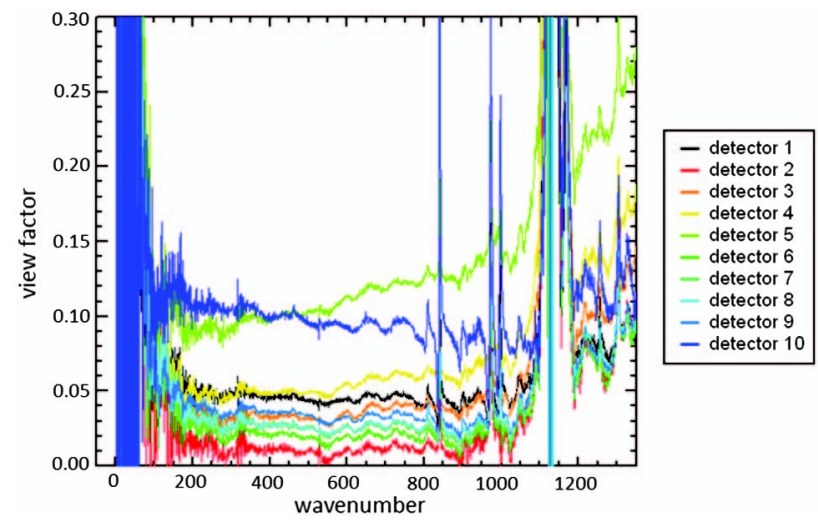

Fig. 8. Fraction of light seen by FIRST coming from the LWIRCS baffle with the window between the scene-select assembly and interferometer dewar removed. should be scaled proportionally with the bolometer DC voltage. When this correction is applied (with no free parameters), the curves seen in Fig. $\underline{9}$ become lines (Fig. 11), and most of the lines are near zero. A line at zero indicates no stray light. As expected, the detectors that show the highest slope and intercept here, 5 and 10, are the ones identified as most sensitive to stray light with the window removed (Fig. 8).

This nonlinearity is unusual in that nonlinearity as a function of the DC level is present, while the spectra are consistent with no nonlinearity across

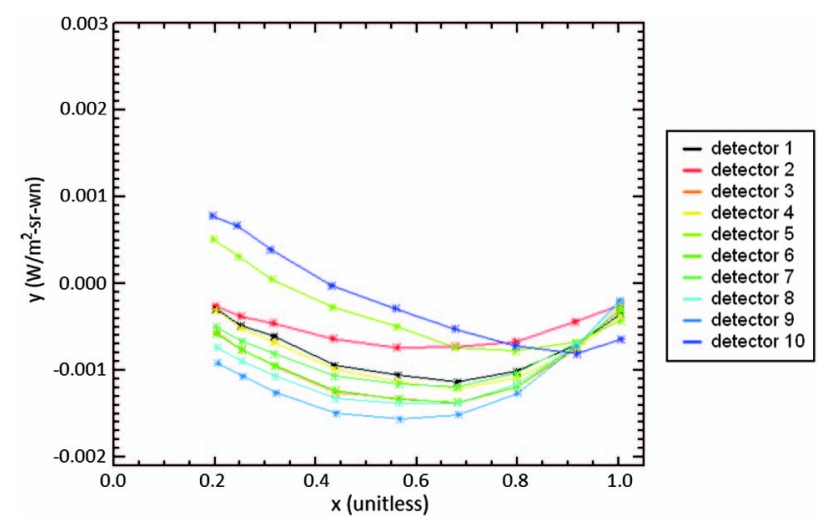

Fig. 9. Plot of $x$ versus $y$ as defined by Eq. (ㅁ) at $400 \mathrm{~cm}^{-1}$ for all detectors. 


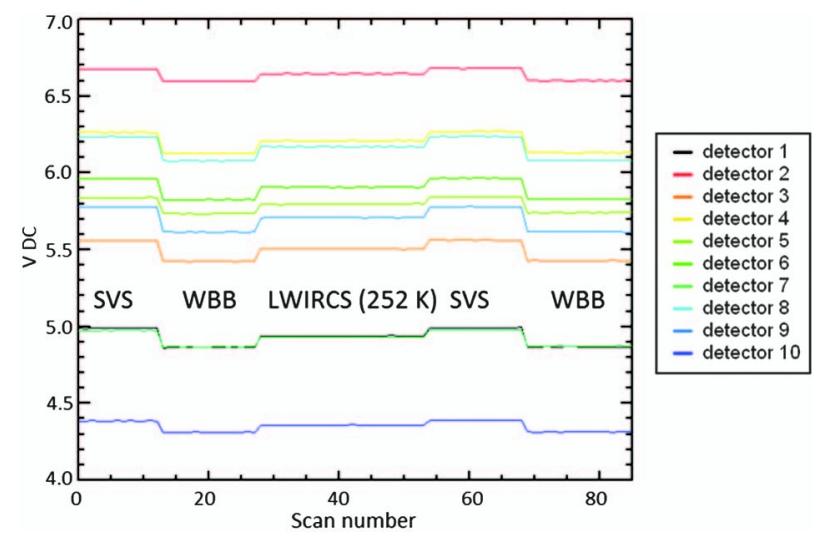

Fig. 10. DC voltage across the FIRST bolometers for all detectors as a function of target viewed.

the interferograms. However, once this effect was found, calculating nonlinearity from the response equation for the bolometer showed that for the FIRST signal levels, there will be detectable nonlinearity that is a function of the DC level on the bolometer, while nonlinearity across the interferogram will be undetectable.

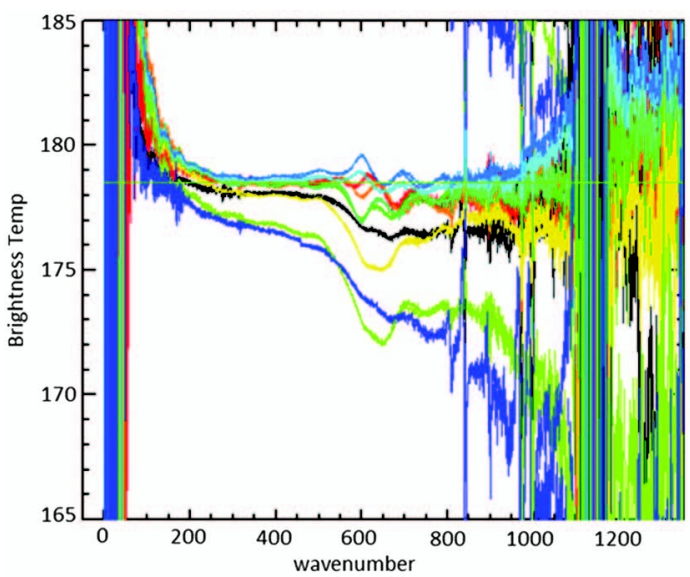

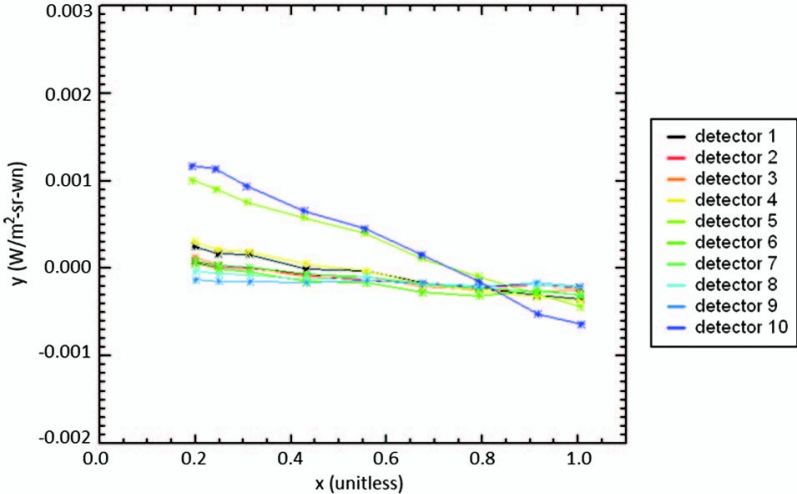

Fig. 11. Plot of $x$ versus $y$ as defined by Eq. (6) at $400 \mathrm{~cm}^{-1}$ for all detectors after nonlinearity correction is applied.

With the linearity correction applied, most of the deviation between the LWIRCS brightness temperature observed by FIRST and the actual LWIRCS temperature is removed, and applying the stray light correction improves it further. Figures 12-15 show this for four LWIRCS temperatures, and the results are similar for all nine LWIRCS temperatures used.

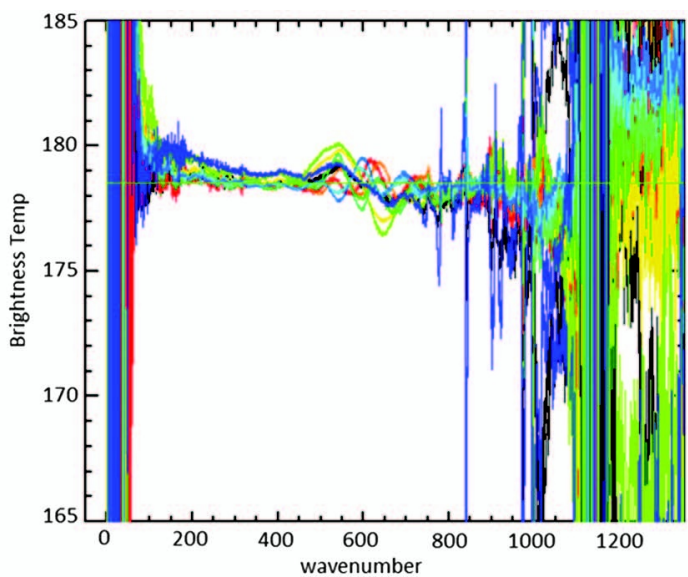

Fig. 12. LWIRCS brightness temperature with LWIRCS at $178.54 \mathrm{~K}$ with the window removed, corrected for nonlinearity (left) and with nonlinearity and stray light corrections (right). The uncorrected data are shown in Fig. $\underline{7}$ upper left.
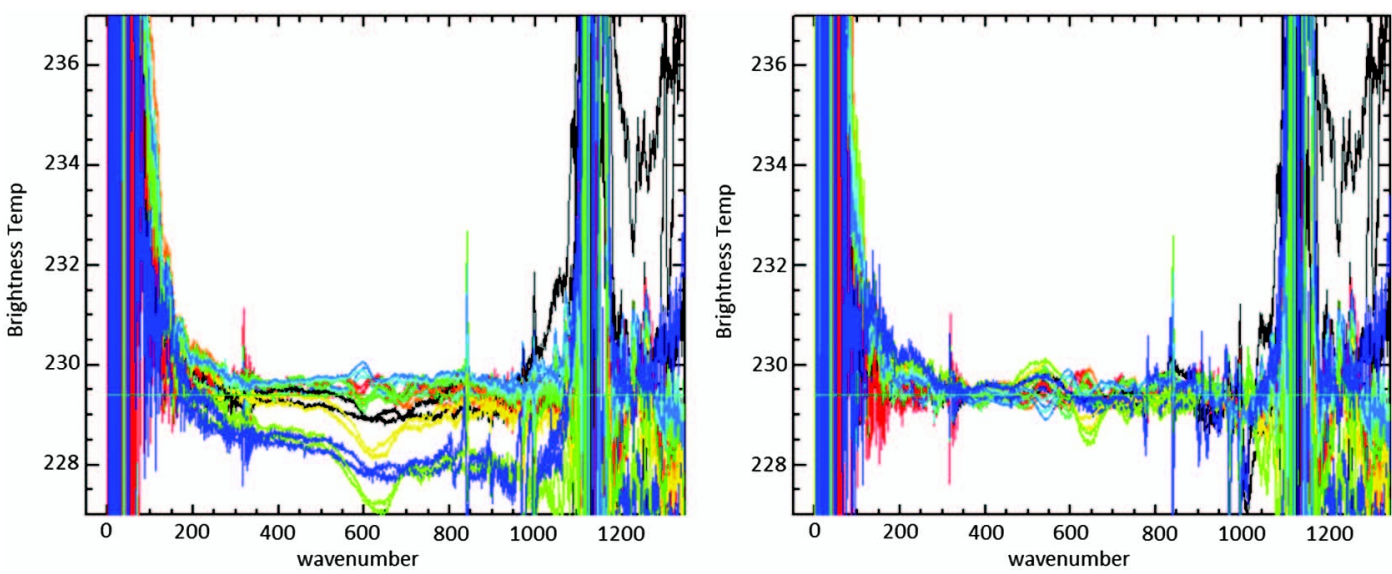

Fig. 13. LWIRCS brightness temperature with LWIRCS at $229.39 \mathrm{~K}$ with the window removed, corrected for nonlinearity (left) and with nonlinearity and stray light corrections (right). The uncorrected data are shown in Fig. 7, upper right. 

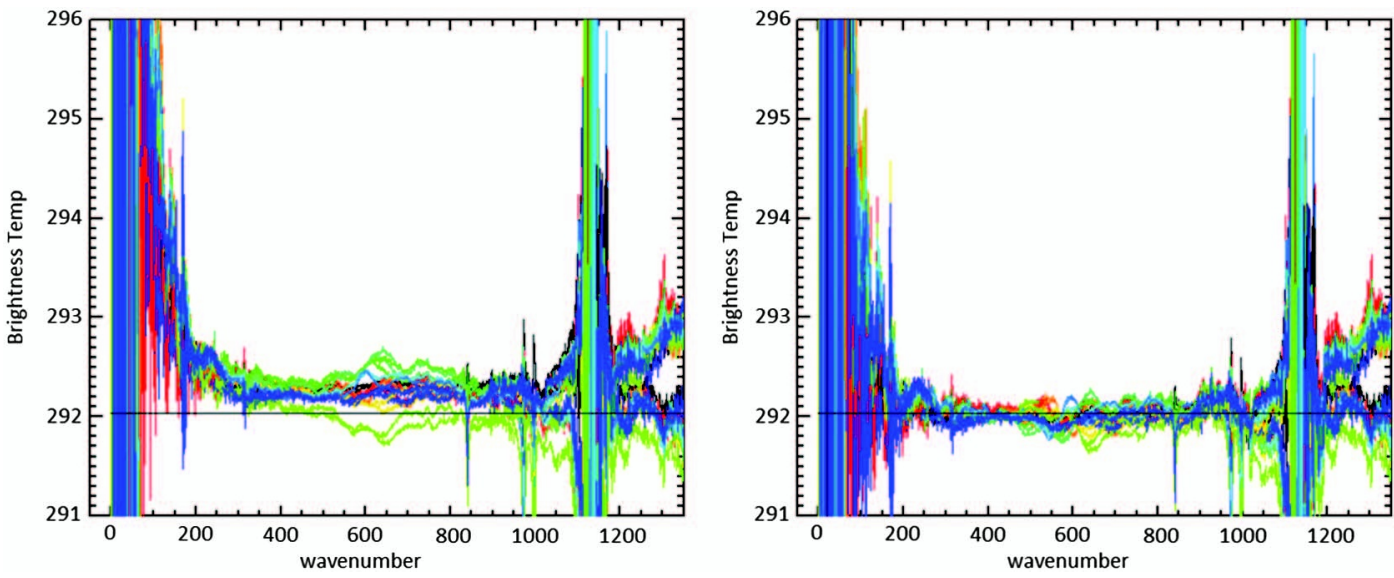

Fig. 14. LWIRCS brightness temperature with LWIRCS at 292.04 K with the window removed, corrected for nonlinearity (left) and with nonlinearity and stray light corrections (right). The uncorrected data are shown in Fig. $\underline{7}$, lower left.
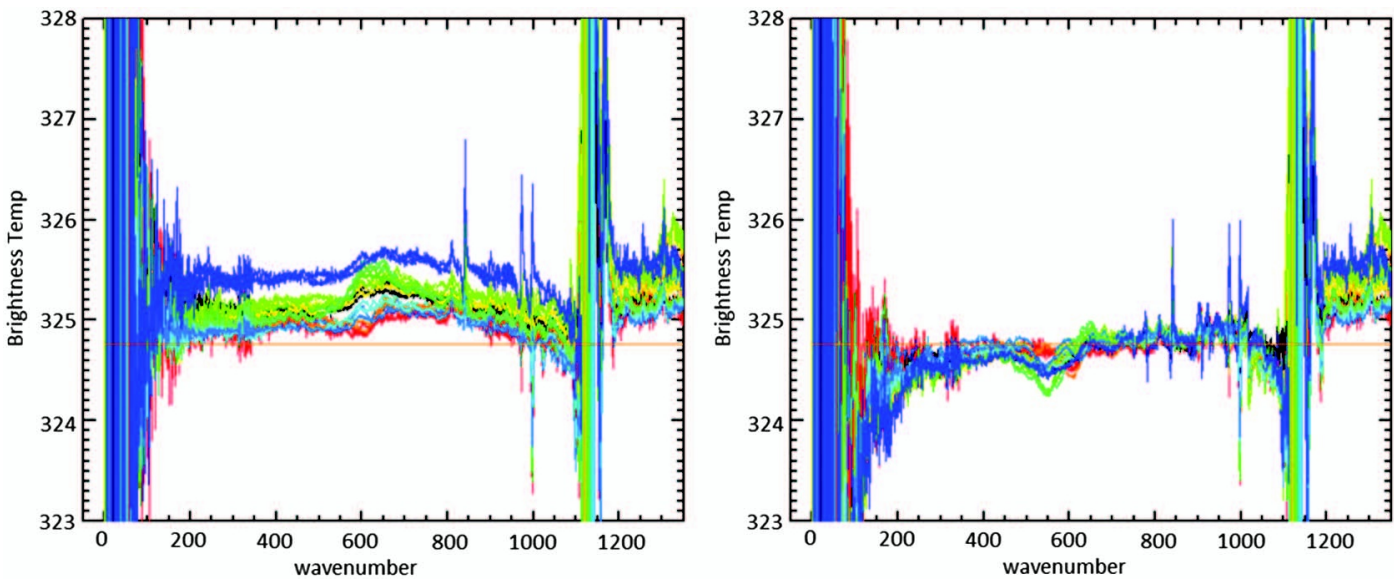

Fig. 15. LWIRCS brightness temperature with LWIRCS at $324.76 \mathrm{~K}$ with the window removed, corrected for nonlinearity (left) and with nonlinearity and stray light corrections (right). The uncorrected data are shown in Fig. 7 , lower right.

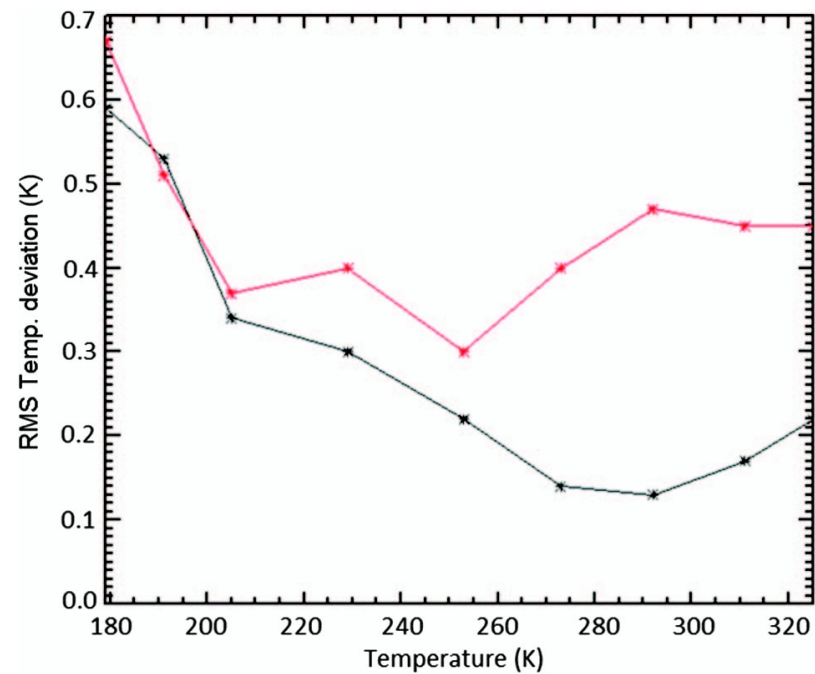

Fig. 16. rms brightness temperature deviation between 200 and $800 \mathrm{~cm}^{-1}$ at all LWIRCS temperatures with window removed, and nonlinearity correction only (red) and nonlinearity plus stray light corrections (black). The curve with the nonlinearity correction only includes data from detectors $2,3,6,7,8$, and 9 and the curve with both corrections includes data from all detectors except 5 and 10 .
Figure 16 shows the rms deviation for all LWIRCS data taken. These data are with the vacuum window removed, but the presence of the window does not significantly change these results. Except for detectors 5 and 10, which are most sensitive to stray light, the nonlinearity correction provides most of the improvement in deviation. For detectors 5 and 10, the stray light correction is more significant as expected. For the remaining detectors, the stray light correction improves deviation significantly only at high temperatures, which shows some stray light is present when viewing the WBB.

\section{Summary and Conclusions}

From Figs. 12-16, the current FIRST absolute accuracy (with the stray light as is and window in place) in a balloon observation mode for detectors $2,3,6,7$, 8 , and 9 is $0.7 \mathrm{~K}$ (rms) to $0.5 \mathrm{~K}$ from 180 to $205 \mathrm{~K}$ and 0.5 to $0.35 \mathrm{~K}$ from 200 to $325 \mathrm{~K}$. Detectors with lower stray light show better performance, and if the stray light were not present, we expect performance of $0.35 \mathrm{~K}$ (one-sigma) or better from 205 to $325 \mathrm{~K}$ and $\sim 0.6 \mathrm{~K}$ down to $180 \mathrm{~K}$. Over most of the range of Earth scene temperatures (205-300 K), the 
expected accuracy is $0.15-0.35 \mathrm{~K}$. Greater improvement is possible, since the stray light correction applied here to derive these values will not exactly match performance in a system designed to suppress stray light.

The results presented here show that the FIRST instrument has some stray light sensitivity, with some detectors being more sensitive than others. Scattering by the vacuum window assembly is a cause, but it is not the only cause. Stray light in FIRST can be further reduced, and future instrument upgrades include plans to do so.

Finally, the cold source calibration shows FIRST does require a bolometer nonlinearity correction, which was made obvious in the cold source calibration data due to the large radiance difference between warm and cold blackbodies. The correction is somewhat unusual in that there is a significant correction proportional to the bolometer DC level with insignificant nonlinearity in the interferograms, but this is consistent with the bolometer response equation and the FIRST signal levels.

The FIRST instrument was deployed to Table Mountain, California, in September 2012 after the warm source calibration [7]. The data from that deployment and from the deployment to Cerro Toco, Chile [6], have been reprocessed to reflect all of the knowledge gained regarding the FIRST instrument performance during the laboratory calibrations. Journal articles describing the science results from both ground-based deployments of FIRST are in preparation. This knowledge will also be extremely useful in developing future space-flight missions such as the proposed Climate Absolute Radiance and Refractivity Observatory [8].

\section{References}

1. M. G. Mlynczak, J. E. Harries, R. Rizzi, P. W. Stackhouse, D. P. Kratz, D. G. Johnson, C. J. Mertens, R. R. Garcia, and B. J. Soden, "Far-infrared: a frontier in remote sensing of Earth's climate, and energy balance," Proc. SPIE 4485, 150-158 (2002).
2. S. A. Clough, M. J. Iacono, and J.-L. Moncet, "Line-by-line calculations of atmospheric fluxes and cooling rates: application to water vapor," J. Geophys. Res. 97, 15761-15785 (1992).

3. C. J. Cox, J. Harries, P. Taylor, P. Green, A. Baran, J. Pickering, A. Last, and J. Murray, "Measurement and simulation of midand far-infrared spectra in the presence of cirrus," Q. J. R. Meteorol. Soc. 136, 718-739 (2010).

4. J. Harries, B. Carli, R. Rizzi, C. Serio, M. Mlynczak, L. Palchetti, T. Maestri, H. Brindley, and G. Masiello, "The far-infrared Earth," Rev. Geophys. 46, RG4004 (2008).

5. M. G. Mlynczak, D. G. Johnson, H. Latvakoski, K. Jucks, M. Watson, D. P. Kratz, G. Bingham, W. A. Traub, S. J. Wellard, C. R. Hyde, and W. Liu, "First light from the far-infrared spectroscopy of the troposphere (FIRST) instrument," Geophys. Res. Lett. 33, L07704 (2006).

6. D. D. Turner, E. J. Mlawer, G. Bianchini, M. P. Cadeddu, S. Crewell, J. S. Delamere, R. O. Knuteson, G. Maschwitz, M Mlynczak, S. Paine, L. Palchetti, and D. C. Tobin, "Groundbased high spectral resolution observations of the entire terrestrial spectrum under extremely dry conditions," Geophys. Res. Lett. 39, L10801 (2012).

7. H. Latvakoski, M. G. Mlynczak, D. G. Johnson, R. P. Cageao, D. P. Kratz, and K. Johnson, "Far-infrared spectroscopy of the troposphere: instrument description and calibration performance," Appl. Opt. 52, 264-273 (2013).

8. B. A. Wielicki, D. F. Young, M. G. Mlynczak, K. J. Thome, S. Leroy, J. Corliss, J. G. Anderson, C. O. Ao, R. Bantges, F. Best, K. Bowman, H. Brindley, J. J. Butler, W. Collins, J. A. Dykema, D. R. Doelling, D. R. Feldman, N. Fox, X. Huang, R. Holz, Y. Huang, Z. Jin, D. Jennings, D. G. Johnson, K. Jucks, S. Kato, D. B. Kirk-Davidoff, R. Knuteson, G. Kopp, D. P. Kratz, X. Liu, C. Lukashin, A. J. Mannucci, N. Phojanamongkolkij, P. Pilewskie, V. Ramaswamy, H. Revercomb, J. Rice, Y. Roberts, C. M. Roithmayr, F. Rose, S. Sandford, E. L. Shirley, W. L. Smith, Sr., B. Soden, P. W. Speth, W. Sun, P. C. Taylor, D. Tobin, and X. Xiong, "Achieving climate change absolute accuracy in orbit," Bull. Am. Meteorol. Soc. 94, 1519-1539 (2013).

9. G. E. Bingham, H. M. Latvakoski, S. J. Wellard, M. G. Mlynczak, D. G. Johnson, W. A. Traub, and K. W. Jucks, "Far-infrared spectroscopy of the troposphere (FIRST): sensor development, and performance drivers," Proc. SPIE 5157, 143-153 (2003).

10. H. Latvakoski and M. Watson, "Performance of highly emissivity 10 to $100 \mu \mathrm{m}$ blackbodies," presented at 2005 CALCON Technical Conference, Logan, Utah, 22-25 August 2005.

11. H. Latvakoski, M. Watson, S. Topham, D. Scott, M. Wojcik, and G. Bingham, "A high-accuracy blackbody for CLARREO," Proc. SPIE 7808, 78080X (2010).

12. J. P. Rice and B. C. Johnson, "The NIST EOS thermal-infrared transfer radiometer," Metrologia 35, 505 (1998).

13. R. L. Richards, "Bolometers for infrared and millimeter waves," J. Appl. Phys. 76, 1-24 (1994). 